\title{
nature
}

\section{Econophysicists matter}

\section{Economics and physics are two disciplines that, contrary to widespread perceptions, have significant common agendas. Shame, then, that the professionals don't do more to recognize the fact.}

$\Lambda$ fter hearing a talk on the application of physics to the social sciences, a physicist in a notoriously traditional department was heard to mutter that it was all very well but it wasn't 'real physics'. It was an article of faith to him that many-body theories in physics could not be applied to animate objects.

Now that it seems clear that bacteria, locusts and even road traffic undergo types of dynamic phase transition, this objection is hard to sustain. But the idea that physics can tell us something about a human system as complex as the economy - that there exists a kind of econophysics - seems harder to swallow.

Would-be econophysicists can therefore find themselves damned from both directions: physicists don't think of the field as physics, whereas economists don't recognize it as their discipline either. Acceptance by the economics community seems a particularly long way off: even fully fledged economists are ostracized by the mainstream if they do not embrace the tenets of 'neoclassical' economic theory, no matter how untenable its principles - identical, utility-maximizing economic agents operating in an equilibrium market - now seem.

The refusal of the economic mainstream to engage with econophysics is lamentable and makes it difficult for physicists to recognize and learn from their mistakes. But it is going to be a fact of life for some time to come. Nevertheless, there is now a body of respected economists who acknowledge the potential value of ideas and tools taken from other sciences, including physics, and who are receptive to the efforts of physicists.

As the News Feature on page 686 illustrates, this opportunity to build links with receptive economists should not be squandered by a lack of quality control in econophysics. Current standards in the field are extremely variable; there is sometimes a sense that physicists are content to find a vaguely plausible way of mapping

some economic question onto a familiar physics-based model, to characterize its behaviour and plot a phase diagram, and leave the matter at that.

This is not particularly good physics; it is certainly poor social science; and it may prove irrelevant to the questions that really matter in understanding economic behaviour. Journals that are willing to publish econophysics must be more vigilant and thorough in their review procedures, and be willing to seek out sympathetically minded economists (who do exist) for advice.

But as some econophysicists argue, it will be hard to improve standards while their efforts are necessarily a sideline tolerated only as long as they also work on 'real' physics (for example,

"The refusal of the economic mainstream to engage with econophysics is lamentable and makes it difficult for physicists to learn from their mistakes." there is not a single chair of econophysics anywhere in the world). This encourages not only the perception but also the reality of a certain dilettantism in the field.

Economics cannot be something for physicists to dabble with. Indeed, the challenge it poses is in some ways even more daunting than that facing physicists who wish to work on biological problems - at least in biology there is a core body of knowledge that, however complex, represents more or less a consensus view. In economics it seems likely that the fundamental principles have yet to be defined, whatever neoclassical theorists say. That is why no single textbook will bring physicists up to speed, and why they have some serious homework to do. They need to be given time to do it.

Econophysics has yet to assemble the critical mass needed to be self-sustaining. If it is not to become extinct, it needs to be made a more attractive career option than it currently is.

\section{Brute force in the clinic}

\section{Clinical microbiologists should catch up with their colleagues and use metagenomics.}

Y ou will not die alone, but with a trillion-strong audience of bacteria and other microscopic buddies. They are glued to our tongues, swarming in our intestines and hitching a ride in our noses. They aid our digestion and shape our immune systems.

Delving into these orifices and their microbial residents is exciting many microbiologists - a sentiment apparent in certain sessions of the American Society for Microbiology meeting in Orlando late last month. Keen to document exactly which microbes are there, many researchers are using brute-force 'metagenomic' studies in which they extract microbial DNA from the body cavity of choice and hurl it all into a sequencing machine. They can identify hundreds or thousands of microbial genes in one swoop.

The discussions in adjacent rooms offered a sharp contrast. Clinical microbiologists - those tasked with identifying the meaner, infectious microbes that invade our bodies and hospitals - use a different set of time-honoured techniques.

Conventionally, they take a swab or sample and attempt to identify the culprit microbe by growing it on certain agar media or examining the shape of a fungi's tentacles under the microscope. Genetic tests are not routine and are typically done to identify one organism at a time. Results can take a day or two to come through. Doctors facing a severe infection use pre-emptive antimicrobial drugs that might later prove fruitless.

Clinical microbiologists have good reasons for sticking with these 
seemingly old-fashioned techniques. Above all, they are foolproof, cheap and definitive - why use an expensive sequencing machine when a culture plate will do? Genetic tests run a high risk of artefacts because one stray contaminating bacterium could produce a false result - and clinical microbiologists have only one precious sample and cannot tolerate errors when a patient's life is at stake.

But used alongside culture methods, the potential rewards of sophisticated genetic analyses are tantalizing. They can reveal a host of bacteria that we cannot grow in culture. Some researchers are designing gene chips that could, if adapted for use in hospitals, reveal which of a range of microbes is there, what strain it is, and which antibiotic-resistance genes and virulence factors it harbours. Used widely, such profiling could help show where a microbe has come from and whether it is spreading.

There is gap to be bridged here. Basic researchers have created ever more crafty ways to hunt down microbes. Clinical microbiologists, who have to deal with hundreds of different diagnostic tests simultaneously, want robust, high-throughput methods that have clear benefits in helping them choose a drug or curtail an infection. As is so often the case, research that converts the former into the latter is in short supply - and neither group has the time or expertise to do it.
More cash for this type of research would, as always, help. It could be used to train more clinical microbiologists in advanced molecular techniques or equip laboratories to trial new diagnostic methods. Meanwhile, basic and clinical researchers can make a simple start, by talking to each other a little more, rather than sitting in separate conference rooms.

Genetic snapshots of the teeming mass of bodily microbes complicate things further. Take, for example, a study released last month into the bane of cystic fibrosis sufferers, $P$ seudomonas aeruginosa (E. E. Smith et al. Proc. Natl Acad. Sci. USA 103, 8487-8492; 2006). By tracking one infection over eight years, the study showed that this bacterium is a moving target: at any one time, many different lineages are present, presumably nestled into different crevices of the lungs. In order to understand and halt an infection, it may not be enough to know that $P$. aeruginos $a$ is there - we might need to know all the different variants and how they interact with each other and with other microbes. This area is ripe for research and might reveal a chink in the microbe's antibiotic-resistant armour.

Metagenomic studies are fascinating for those of us grown-ups still childishly interested in our own dank, dark recesses. But microbial surveys have urgent application in medical care. The research and medical community must ensure they are used there.

\section{Peer review on trial}

\section{Introducing two Nature initiatives.}

7 his publication champions the value of editorially driven or editorially selected content. But we are always keen to try new things, and we are now experimenting around the edges of that principle, to make the most of online interactivity.

In recent months we have started attaching blogs to every daily news item on our news@nature.com website; see, for example, a debate on the 'missing link' Tiktaalik roseae (http://blogs.nature.com/ news/blog/2006/04/the_fish_that_crawled_out_of_t.html).

Blogs are unlikely to replace journalism, but are probably here to stay as a valuable complement. Less certain is the outcome of a trial that we launch this week: a test of a particular type of open peer review. The trial is accompanied by a general online debate about peer review; see www.nature.com/nature/peerreview/index.html.

During the trial, which will last several months, Nature's traditional approach to peer review will continue: typically, we send selected submissions to two or three experts whose identities are kept confidential. We believe that this approach works well. Meanwhile, over the next few weeks, the web debate will explore other approaches, as well as the potential for online techniques to unpack the various functions of conventional journals, the ethics of peer review, and more.

Our online trial opens up a parallel track of peer review for submitted papers for authors willing to go down that route. The traditional process will still be applied to all submissions selected for peer review. But we will also offer to post the submitted manuscript onto an open website. Anyone can then respond to it by posting online comments, provided they are willing to sign them. Once
Nature's editors have received all the comments from their solicited confidential reviewers, the open website will cease to take comments, and all the opinions will be considered by the editors as well as the authors.

The open procedure will only be applied to the first version of a manuscript. Once a manuscript has been accepted or rejected, the editor will privately assess the value of each open contribution.

The trial will run for several months. Once all final decisions on the relevant manuscripts have been reached, we will conduct an aggregated assessment of the comments received as well as the work required to do justice to them. We will report the result in these pages, and consider whether our peer-review procedures should be adapted appropriately. We expect any changes to be complementary to our existing processes, not a replacement.

We use the word 'trial' rather than 'experiment' advisedly. An experiment would set out to establish the fundamental benefits and disadvantages of this
"Our onlinetrial opensup a paralleltrack of peer review for submitted papers for authors willing togo down that route." approach to open peer review. We do not daim such grand ambitions. We simply want to suck it and see, and a survey of recent authors has demonstrated enough interest in the idea to make such a trial worthwhile.

Anypaper that we post on our open website for comments will of course be fair game for public access and for journalists. Prior media coverage will not endanger their acceptance. But journalists will be aware that papers not subjected to peer review carry their own risks.

Here, as in other ways, Nature and its publishers are exploring and expanding our opportunities online. But our core goal remains as always: to bring our readers the most stimulating content that our editorial skills can deliver. 